\title{
Recurrence quantification analysis for the identification of burst phase synchronisation
}

E. L. Lameu, S. Yanchuk, E. E. N. Macau, F. S. Borges, K. C. Iarosz, I. L. Caldas, P. R. Protachevicz, R. R. Borges, R. L. Viana, J. D. Szezech, A. M. Batista, and J. Kurths

Citation: Chaos 28, 085701 (2018); doi: 10.1063/1.5024324

View online: https://doi.org/10.1063/1.5024324

View Table of Contents: http://aip.scitation.org/toc/cha/28/8

Published by the American Institute of Physics

\section{Articles you may be interested in}

Optimizing the detection of nonstationary signals by using recurrence analysis

Chaos: An Interdisciplinary Journal of Nonlinear Science 28, 085703 (2018); 10.1063/1.5022154

Introduction to focus issue: Recurrence quantification analysis for understanding complex systems

Chaos: An Interdisciplinary Journal of Nonlinear Science 28, 085601 (2018); 10.1063/1.5050929

Phase space reconstruction for non-uniformly sampled noisy time series

Chaos: An Interdisciplinary Journal of Nonlinear Science 28, 085702 (2018); 10.1063/1.5023860

Analysis of diagonals in cross recurrence plots between heart rate and systolic blood pressure during supine position and active standing in healthy adults

Chaos: An Interdisciplinary Journal of Nonlinear Science 28, 085704 (2018); 10.1063/1.5024685

Sleep-wake detection using recurrence quantification analysis

Chaos: An Interdisciplinary Journal of Nonlinear Science 28, 085706 (2018); 10.1063/1.5024692

Extended recurrence plot and quantification for noisy continuous dynamical systems

Chaos: An Interdisciplinary Journal of Nonlinear Science 28, 085722 (2018); 10.1063/1.5025485

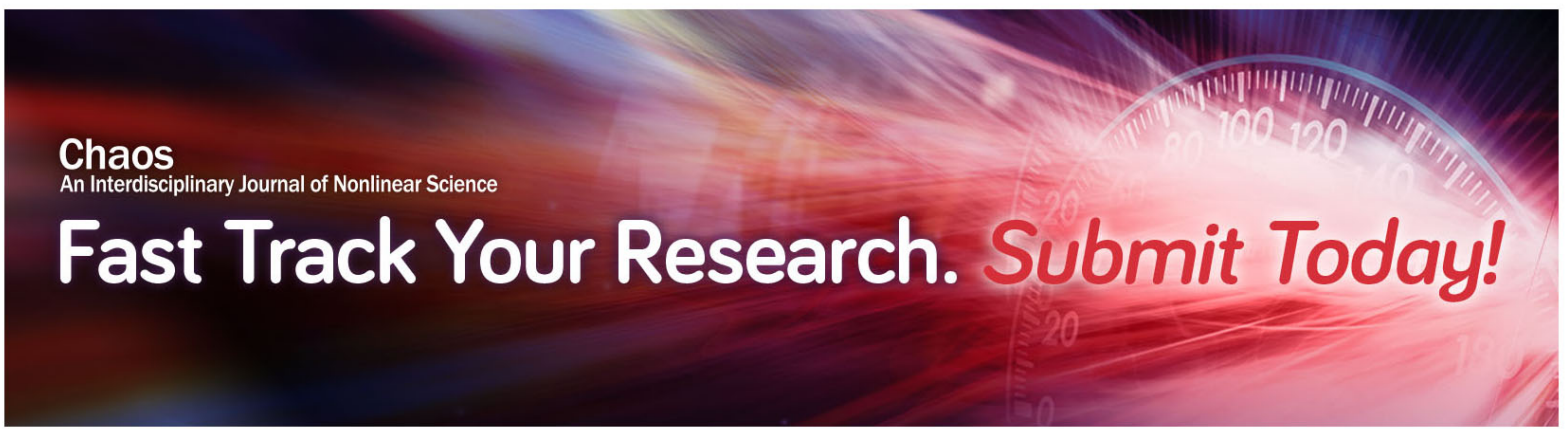




\title{
Recurrence quantification analysis for the identification of burst phase synchronisation
}

\author{
E. L. Lameu, ${ }^{1,2}$ S. Yanchuk, ${ }^{3}$ E. E. N. Macau, ${ }^{1,4}$ F. S. Borges, ${ }^{5}$ K. C. Iarosz,,${ }^{2,6,7,8}$ I. L. Caldas, ${ }^{6}$ \\ P. R. Protachevicz, ${ }^{9}$ R. R. Borges, ${ }^{10}$ R. L. Viana, ${ }^{11}$ J. D. Szezech, Jr., ${ }^{9,12}$ A. M. Batista, ${ }^{6,7,8,9,12}$ and \\ J. Kurths ${ }^{2,7}$ \\ ${ }^{1}$ National Institute for Space Research, São José dos Campos, São Paulo 12227-010, Brazil \\ ${ }^{2}$ Department of Physics, Humboldt University, Berlin 12489, Germany \\ ${ }^{3}$ Institute of Mathematics, Technical University of Berlin, Berlin 10623, Germany \\ ${ }^{4}$ ICT-Institute of Science and Technology, Federal University of São Paulo, São José dos Campos, São Paulo 12231-280, Brazil \\ ${ }^{5}$ Center for Mathematics, Computation, and Cognition, Federal University of ABC, São Bernardo do Campo, São Paulo \\ 09606-045, Brazil \\ ${ }^{6}$ Institute of Physics, University of São Paulo, São Paulo 05508-900, Brazil \\ ${ }^{7}$ Potsdam Institute for Climate Impact Research, Potsdam 14473, Germany \\ ${ }^{8}$ Institute for Complex Systems and Mathematical Biology, University of Aberdeen, SUPA, Aberdeen AB24 3UE, United Kingdom \\ ${ }^{9}$ Program of Post-graduation in Science, State University of Ponta Grossa, Ponta Grossa, Paraná 84030-900, Brazil \\ ${ }^{10}$ Department of Mathematics, Federal University of Technology-Paraná, Ponta Grossa, Paraná 84016-210, Brazil \\ ${ }^{11}$ Department of Physics, Federal University of Paraná, Curitiba, Paraná 80060-000, Brazil \\ ${ }^{12}$ Department of Mathematics and Statistics, State University of Ponta Grossa, Ponta Grossa, Paraná 84030-900, Brazil
}

(Received 31 January 2018; accepted 22 March 2018; published online 21 August 2018)

\begin{abstract}
In this work, we apply the spatial recurrence quantification analysis (RQA) to identify chaotic burst phase synchronisation in networks. We consider one neural network with small-world topology and another one composed of small-world subnetworks. The neuron dynamics is described by the Rulkov map, which is a two-dimensional map that has been used to model chaotic bursting neurons. We show that with the use of spatial RQA, it is possible to identify groups of synchronised neurons and determine their size. For the single network, we obtain an analytical expression for the spatial recurrence rate using a Gaussian approximation. In clustered networks, the spatial RQA allows the identification of phase synchronisation among neurons within and between the subnetworks. Our results imply that RQA can serve as a useful tool for studying phase synchronisation even in networks of networks. Published by AIP Publishing. https://doi.org/10.1063/1.5024324
\end{abstract}

One of the most relevant phenomena observed in various coupled systems is synchronisation. There are experimental evidences of synchronisation, e.g., in power grids, electronic circuits, and neural networks. With this in mind, we investigate methods for identifying synchronous behaviour in coupled systems with burst dynamics. Burst is a dynamic behaviour where signals are fired with high frequency. We build a network composed of bursting neurons by means of coupled Rulkov maps not only in single networks but also in a network of networks which mimics better the brain topology. The study of neural synchronisation is important due to the fact that it can be related not only to memory processes but also to pathological conditions, where abnormal neural synchronisation can lead to neurological disorders. Recurrence quantification analysis (RQA) is a method of nonlinear data analysis that has been used to investigate dynamical systems. We show that RQA can be used successfully as a diagnostic tool to study the transition to synchronisation in neural bursting networks.

\section{INTRODUCTION}

Neural synchronisation has been observed during different tasks, at rest, and within different frequency bands. ${ }^{1}$ Womelsdorf et al. $^{2}$ suggested that synchronisation has relevant consequences for neural interactions, contributing to cognitive functions. Nevertheless, synchronous behaviour in the brain is also related to neurological disorders. ${ }^{3}$ Motor symptoms of Parkinson's disease are associated with synchronised oscillatory activity in some parts of the brain. ${ }^{4}$ Similarly, epileptic seizures are related to abnormal synchronised firing of neurons. ${ }^{5}$

In this work, we study synchronisation in neural networks of coupled Rulkov maps. We consider two cases: the first is a single small-world network and the second is a network of networks ${ }^{6,7}$ which mimics better the brain topology. The Rulkov map ${ }^{8}$ is a two-dimensional iterated map developed to model neurons with burst dynamics. We analyse a topology according to small-world property because it exhibits high levels of clustering and short path lengths. ${ }^{9}$ There are many reports about the existence of small-world brain functional networks. ${ }^{10,11}$ Lameu et al. ${ }^{12}$ observed bursts synchronisation in the cat cerebral cortex modelled by means of coupled smallworld networks of Rulkov neurons. They also studied the effects of perturbations on the cat cerebral cortex to suppress burst synchronisation. ${ }^{13}$

We apply RQA as a very efficient alternative method to identify phase synchronisation in the two networks considered here. The RQA was developed in the late 1980s. ${ }^{14}$ Santos et al. used RQA to identify chimera states and chimera collapse in coupled dynamical systems ${ }^{15}$ and neural network. ${ }^{16}$ Recurrence plots have been used as a numerical tool to study 
time series of dynamical systems. ${ }^{17}$ The recurrence matrix is obtained by comparing distances between different points on a trajectory in the phase space. If the distance is smaller than a specific threshold, the corresponding points are considered to be recurrent and the corresponding matrix element is set to one (otherwise it is zero). For example, recurrence plots show whether a system possesses periodic, chaotic, or stochastic behaviour. Vasconcelos et al. ${ }^{18}$ applied an extension of the usual recurrence concept to study spatial disorder in a network at a fixed time. They applied the laminarity and trapping length ${ }^{19}$ concepts to observe synchronisation in a lattice of logistic maps. In this work, our main intention is to show that a new measure inspired by laminarity, we call as laminarity inspired, and recurrence rate can be used as diagnostic tools of neural phase synchronisation.

This paper is organised as follows: Sec. II introduces the Rulkov map. Section III defines the order parameter and the spatial recurrence quantification analysis. In Sec. IV, we apply the spatial RQA to a neural network with small-world properties to identify neural phase synchronisation. In Sec. V, we use the collective dynamics measurement in a network of networks, where the subnetworks have small-world properties. Section VI concludes the article.

\section{BURSTING NEURAL MODEL}

The model proposed by Rulkov ${ }^{8}$ is a model for a neuron generating spiking and bursting activity, and it is given by the two-dimensional iterated map

$$
\begin{aligned}
& x_{n+1}=\frac{\alpha}{1+x_{n}^{2}}+y_{n}, \\
& y_{n+1}=y_{n}-\sigma x_{n}-\beta,
\end{aligned}
$$

where $n$ is the discrete time, $x_{n}$ is the fast dynamical variable that represents the membrane potential, and $y_{n}$ is the slow dynamical variable. The nonlinearity parameter $\alpha$ is responsible for the spiking timescale, and the small parameters $\sigma$ and $\beta$ describe the slow timescale. In our simulations, we consider $\sigma=\beta=0.001, \alpha$ uniformly distributed in the interval $[4.1,4.4]$, resulting in non-identical neurons, and initial conditions $x_{0}$ and $y_{0}$ randomly distributed in the interval $[-1,1]$.

In Figs. 1(a) and 1(b), we see that the time evolution of $x_{n}$ and $y_{n}$ exhibits bursts and regular saw-tooth oscillations, respectively. A burst starts when $y_{n}$ has a local maximum in a well-defined instant of time $n_{k}$. The time interval of a burst is given by $n_{k+1}-n_{k}$ (Fig. 1). We can associate the phase with the time evolution of the bursts,

$$
\phi_{n}=2 \pi \frac{n-n_{k}}{n_{k+1}-n_{k}},
$$

where $k$ is an integer.

\section{COLLECTIVE DYNAMICS MEASUREMENT}

\section{A. Order parameter}

Synchronisation ${ }^{20,21}$ can be measured by the Kuramoto order parameter, ${ }^{22}$ applied in different kinds of synchronous behaviours, such as power-grids, ${ }^{23-25}$ Hamiltonian systems, ${ }^{26}$

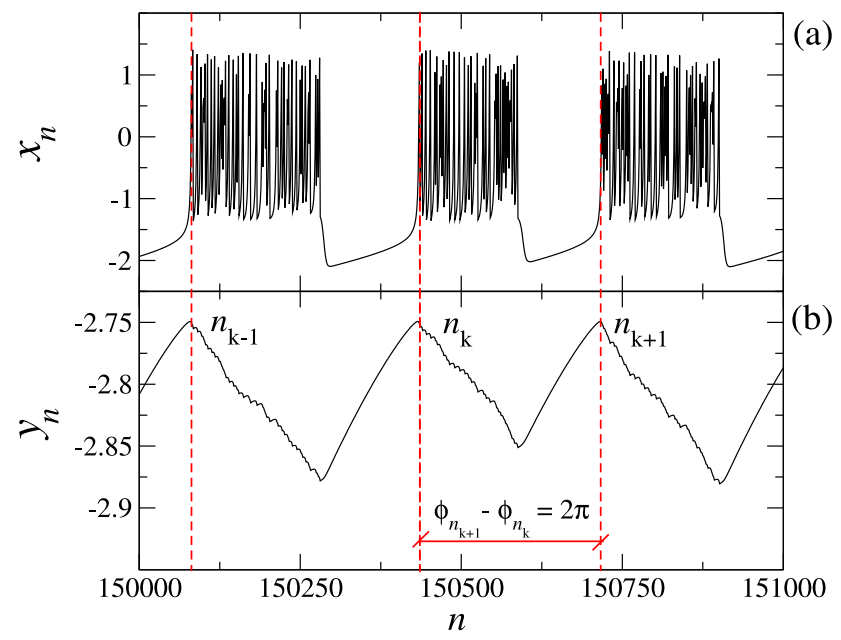

FIG. 1. Time evolution $n$ of the (a) fast $x_{n}$ and (b) slow $y_{n}$ variables in the Rulkov map, where $n_{k}$ denotes when the neural burst starts.

and neural networks. ${ }^{27}$ The order parameter is defined as

$$
r_{n} \mathrm{e}^{\mathrm{i} \Phi_{n}}=\frac{1}{N} \sum_{i=1}^{N} \mathrm{e}^{i \phi_{n}^{(i)}},
$$

where $r_{n}$ and $\Phi_{n}$ are the amplitude and the angle of a centroid phase vector, respectively, and $N(i=1,2, \ldots, N)$ is the total number of neurons. The amplitude of $r_{n}$ ranges from 0 to 1 . For desynchronised phases, one finds $r_{n} \ll 1$, namely, the phases of the bursts are out of synchrony. When $r_{n}=1$, all phases are identical, i.e., the bursts are synchronised. We calculate the Kuramoto order parameter in order to compare with RQA and to show that RQA provides substantial complementary information, such as patterns of synchronised structures.

\section{B. Recurrence quantification analysis}

The recurrence concept was presented by Eckmann et al. ${ }^{17}$ through the recurrence plot (RP) of dynamical systems. Zbilut and Webber ${ }^{28}$ introduced recurrence quantification analysis (RQA) which ${ }^{14}$ has been used as a tool for the study of many nonlinear dynamical systems, e.g., the exploration of cardiac signals ${ }^{29}$ or electrostatic fluctuations in fusion plasma. ${ }^{30}$ We identify neural phase synchronisation by means of methods based on spatial recurrence rate and laminarity inspired.

The spatial recurrence rate $\mathrm{RR}$ is defined as

$$
\mathrm{RR}_{n}=\frac{1}{N^{2}} \sum_{i, j=1}^{N} \mathbf{R}_{i, j}(n),
$$

where $n$ corresponds to the time and the $\mathbf{R}_{i, j}(n)$ is the $N \times N$ recurrence matrix at time $n$. $\mathbf{R}_{i, j}(n)$ is given by

$$
\mathbf{R}_{i, j}(n)=\Theta\left(l-\left|\phi_{n}^{(i)}-\phi_{n}^{(j)}\right|\right),
$$

where $l$ is the threshold distance, $\Theta(\cdot)$ is the Heaviside function, and $\phi_{n}^{(j)}$ is the phase of neuron $j$ at time $n$. The nonzero elements of the spatial recurrence matrix $\mathbf{R}_{i, j}(n)$ at a given time $n$ represent recurrent phases, namely, the pairs of neurons which have phase differences smaller than the threshold $l$. 
In our approach, $\mathrm{RR}_{n}$ varies in the interval $[0,1]$ and gives information about the proportion of synchronised pairs of neurons in the network. We are also interested in verifying whether pairs of synchronised neurons are isolated or organised in groups. To do that, we use the laminarity inspired

$$
\mathrm{L}_{n}=\frac{\sum_{v=v_{\min }}^{N} v P(v)}{\sum_{v=1}^{N} v P(v)},
$$

where $P(v)$ is the number of columns in $\mathbf{R}_{i, j}(n)$ with exactly $v$ nonzero elements, normalised by $N$. The laminarity inspired $\left(\mathrm{L}_{n}\right)$ is the ratio between the number of oscillator pairs belonging to synchronised groups of size $v \geq v_{\min }$ and the total number of synchronised pairs [points in $\mathbf{R}_{i, j}(n)$ ]. We choose $v_{\min }=l N / 2$, while a more detailed explanation for this choice will be given in Sec. IV B. Through the RP, we also calculate the average size of the structures $\mathrm{S}$,

$$
\mathrm{S}_{n}=\frac{\sum_{v=v_{\min }}^{N} v P(v)}{N \sum_{v=v_{\min }}^{N} P(v)} .
$$

\section{SMALL-WORLD NETWORK}

\section{A. Numerical results}

Coupled dynamical systems have been used to describe behaviours of neural networks. There are experimental evidences that some anatomical connections in the brain have small-world properties. ${ }^{10,11}$ We consider a small-world topology formulated by Newman and Watts, ${ }^{31}$ where a regular network with periodic boundary conditions [Fig. 2(a)] is transformed into a small-world network [Fig. 2(b)] adding shortcuts. Figure 2(c) shows the adjacency matrix for such a network with $N=200$ neurons, where the black points indicate the pairs of connected neurons. All neurons are connected locally with the first two neighbours [Fig. 2(a)], and then shortcuts are randomly chosen with probability $p=0.1$ [Fig. 2(b)].

We consider a mean-field coupling among the connected neurons,

$$
\begin{aligned}
& x_{n+1}^{(i)}=\frac{\alpha^{(i)}}{1+\left(x_{n}^{(i)}\right)^{2}}+y_{n}^{(i)}+\frac{\varepsilon}{k^{(i)}} \sum_{j \in I} x_{n}^{(j)}, \\
& y_{n+1}^{(i)}=y_{n}^{(i)}-\sigma x_{n}^{(i)}-\beta,
\end{aligned}
$$

where $\varepsilon$ is the coupling strength and each neuron $i$ is coupled with a set $I$ comprising $k^{(i)}$ other neurons in the network with $N$ neurons.

Figures 3(a)-3(c) show the spatial RPs for $\varepsilon=0$, $\varepsilon=0.03$, and $\varepsilon=0.1$, respectively, at the time $n=150000$. Considering $l=0.1$ and increasing the $\varepsilon$ value, the number of black dots and vertical structures in the RP increases due to the phase synchronisation among the neurons. In the corresponding figures, increasing $\varepsilon$, the phase distribution changes from a uniform [Fig. 3(d)] to a unimodal distribution with a decreasing standard deviation [see Figs. 3(e) and 3(f)]. The solid line in Figs. 3(d)-3(f) shows a fit of the phase distributions to a Gaussian one. The possibility of a good fit of the
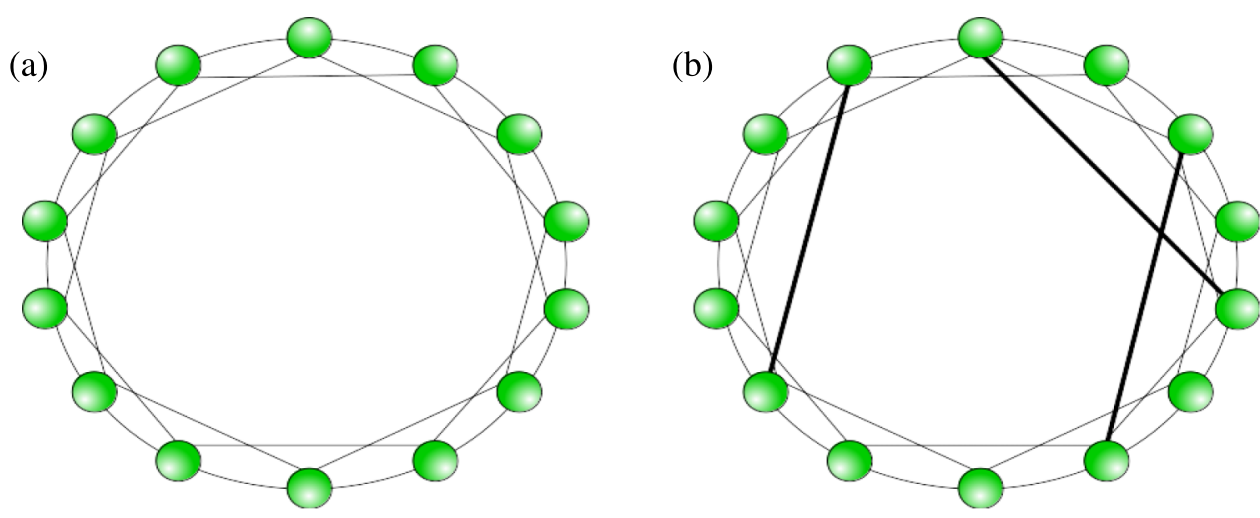

(c)

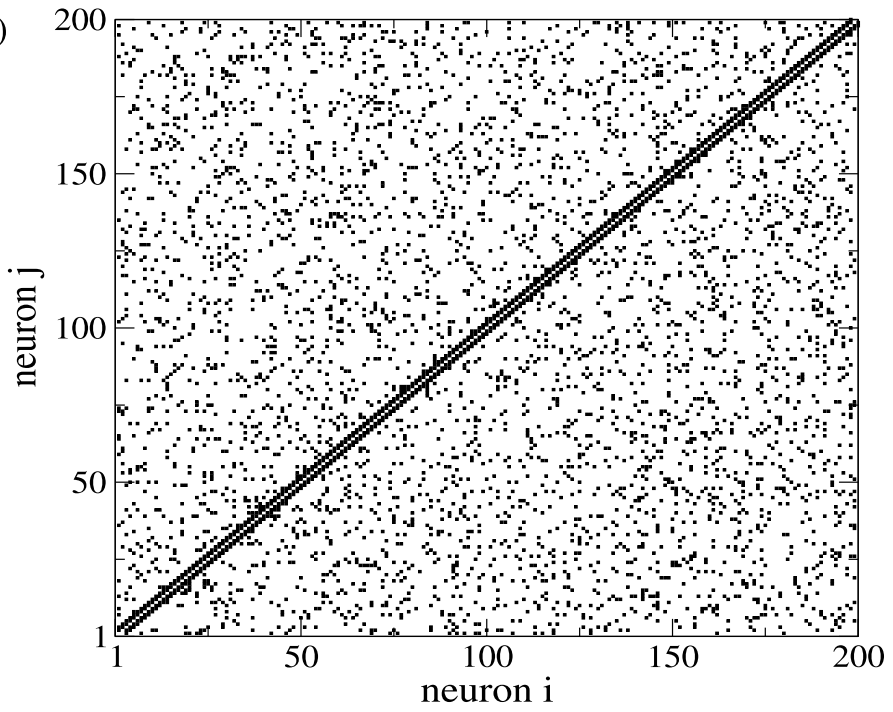

FIG. 2. Schematic representations of (a) regular and (b) small-world networks. (c) The adjacency matrix of our network with small-world properties, where the black points indicate the pairs of connected neurons. 
data by a Gaussian distribution allows for an analytical study of $r$ and RR, as described in Sec. IV B.

We also calculate the time evolution of the quantities $r_{n}, \mathrm{RR}_{n}, \mathrm{~L}_{n}$, and $\mathrm{S}_{n}$ for $\varepsilon=0, \varepsilon=0.03$, and $\varepsilon=0.1$, as shown in Figs. 3(g)-3(i), respectively. For the uncoupled neurons $(\varepsilon=0)$, all the RQA measurements have small values, showing no synchronous behaviour, and in accordance with the small $r_{n}$ values. For $\varepsilon=0.03, \mathrm{RR}_{n}$ exhibits that about $15 \%$ of the neuron pairs are recurrent, and the increasing of $\mathrm{L}_{n}$ indicates that more than $80 \%$ of them are part of a synchronised group of neurons. However, as shown by $\mathrm{S}_{n}$, the sizes of these groups are small and oscillate around 0.15 . Therefore, for $\varepsilon=0.03$ and $l=0.1$, through the RQA, it is possible to observe the presence of a large amount of small synchronised neural groups. The $r_{n}$ also display synchronisation in the network, but it does not give any information about synchronised groups. Increasing $\varepsilon$ to $0.1, r_{n}$ and $\mathrm{L}_{n}$ have values near unity due to the wide-spread synchronisation. The values of $\mathrm{RR}_{n}$ and $\mathrm{S}_{n}$ also increase showing that there are more synchronised pairs of neurons and larger groups.
In Fig. 4, we calculate the time average of the collective dynamics measurements as a function of $\varepsilon$ for different $l$ values, where we consider a time window equal to 50000 iterations. Figure 4(a) exhibits the dependence of $\overline{\mathrm{RR}}$ on $l$ and gives us an average proportion of synchronised pairs of neurons for a $\varepsilon$ value. $l$ provides us a way to adjust the RQA to identify synchronised and desynchronised behaviours. We see that $\overline{\mathrm{L}}$ has almost the same behaviour for different $l$ values, which occurs because the $v_{\min }$ changes with $l$ [Fig. 4(b)]. For $\bar{S}$, we observe that for larger $l$ more recurrent points are computed, and as a consequence larger groups of synchronised neurons can be identified.

We also calculate the probability distribution of $\mathrm{S}$, namely, the probabilities of occurrence of different possible average structure sizes in the spatial RP. Figure 5 displays $\varepsilon$ versus $\mathrm{S}$, where the colour bar represents the probability distribution $\mathrm{P}(\mathrm{S})$. Considering $l=0.1$, small structures are found for $\varepsilon<0.025$, while larger groups are observed for $\varepsilon>0.15$. For each $\varepsilon$ value, there is an $S$ interval with $\mathrm{P}(\mathrm{S})>0$, namely, it is possible to find different average sizes. (a)

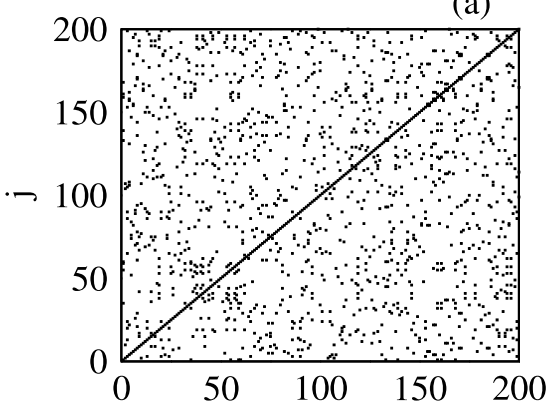

(b)

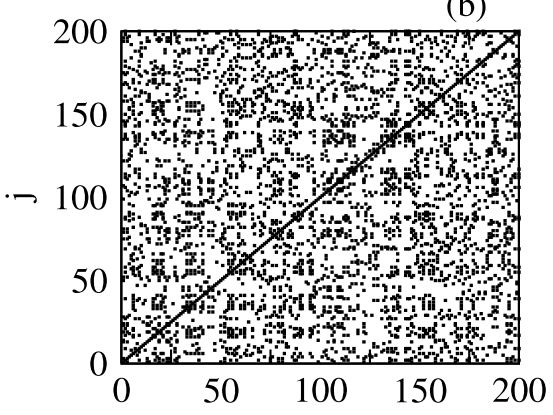

(c)

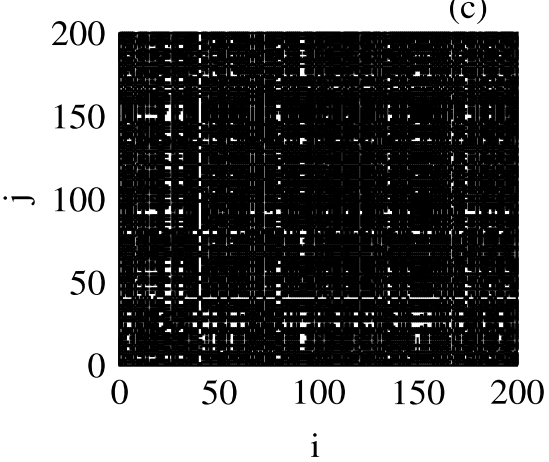

(d)

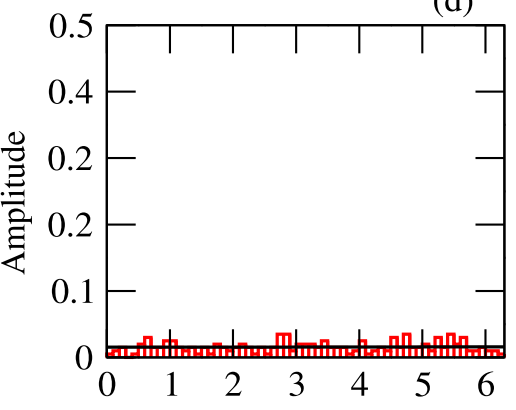

(e)

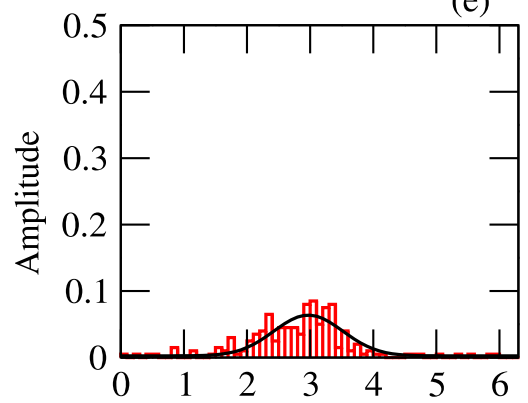

(f)

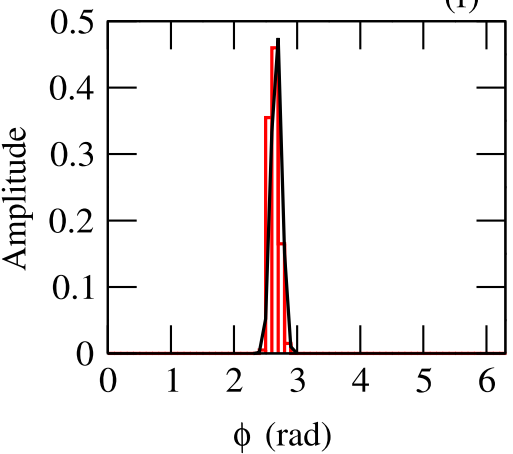

(g)

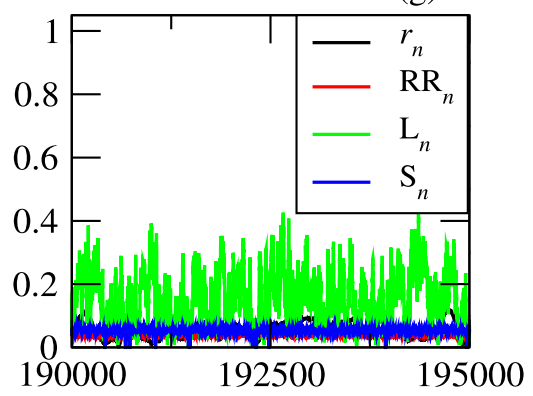

(h)

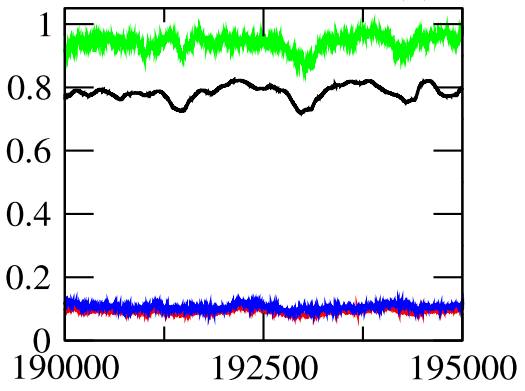

(i)

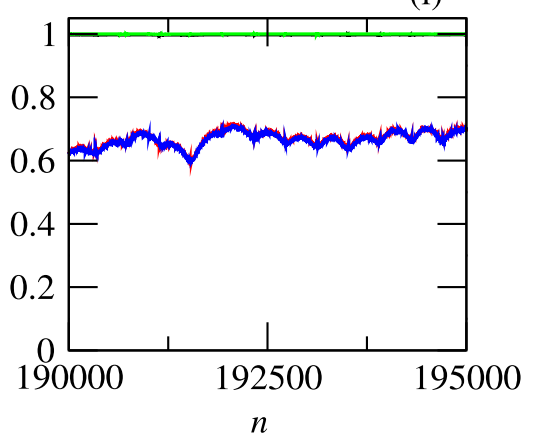

FIG. 3. RPs for $N=200, p=0.1, l=0.1$, (a) $\varepsilon=0$, (b) $\varepsilon=0.03$, and (c) $\varepsilon=0.1$. Panels (d), (e), and (f) show the respective $\phi$ distributions. In panels (g), (h), and (i), we calculate the respective collective dynamics measurements: $r_{n}$ (black), $\mathrm{RR}_{n}$ (red), $\mathrm{L}_{n}$ (green), and $\mathrm{S}_{n}$ (blue). 

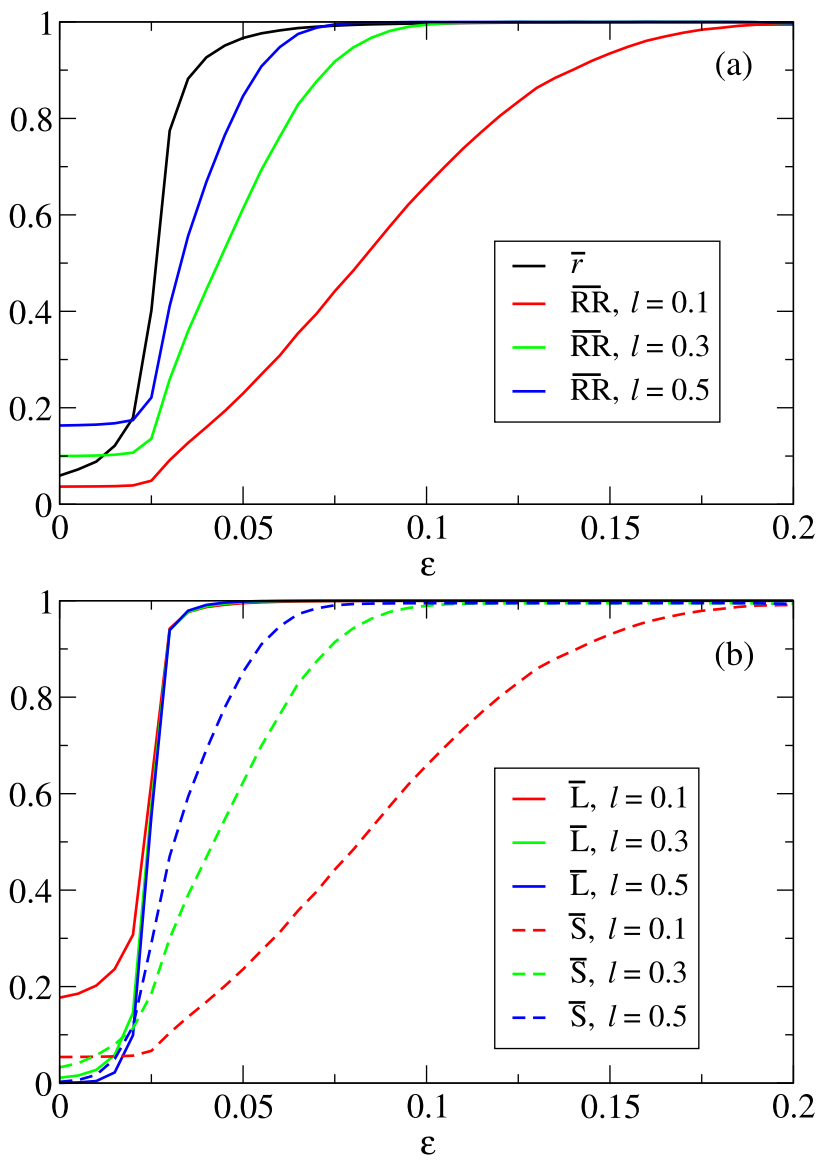

FIG. 4. (a) $\bar{r}$ and $\overline{\mathrm{RR}}$ as a function of $\varepsilon$ for $l=0.1$ (red), $l=0.3$ (green), and $l=0.5$ (blue). (b) $\overline{\mathrm{L}}$ and $\overline{\mathrm{S}}$ as a function of $\varepsilon$ for different $l$ values.

The values of $\mathrm{L}$ and $\mathrm{S}$ are independent of the reordering of the nodes. This occurs due to the fact that the vertical structures are calculated considering only the amount of non-zero elements in each column, i.e., we do not take into account the gaps between them (the zero elements).

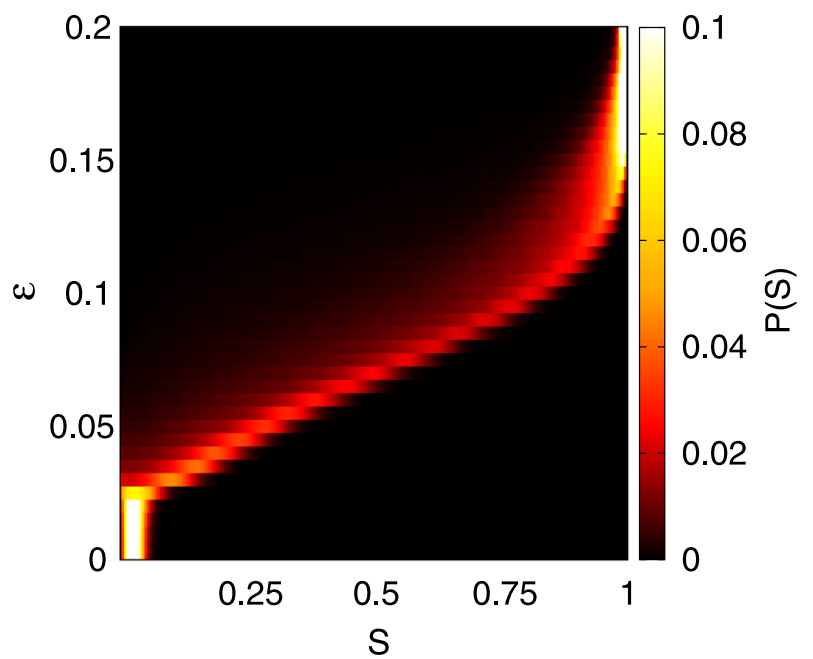

FIG. 5. $\varepsilon \times \mathrm{S}$ for $l=0.1$, where the colour bar corresponds to size probability $\mathrm{P}(\mathrm{S})$.

\section{B. Analytical estimations of spatial recurrence rate for normally distributed phases}

As numerics in Figs. 3(d)-3(f) indicates the phase distributions for the single small-world network for different couplings can be approximated with a normal distribution. Therefore, let us assume that the oscillator phases are distributed accordingly to a circular normal (von Mises) distribution

$$
\rho_{\kappa}(\phi)=\frac{1}{2 \pi I_{0}(\kappa)} \exp [\kappa \cos (\phi-\mu)],
$$

where $I_{0}(\kappa)$ is the modified Bessel function of order $0, \mu$ is the mean, which we assume to be zero without loss of generality, and $\kappa$ is the parameter measuring the degree of synchronisation. The order parameter corresponding to the distribution (9) can be computed as ${ }^{32}$

$$
r(\kappa)=\int_{0}^{2 \pi} e^{i \phi} \rho_{\kappa}(\phi) d \phi=\frac{I_{1}(\kappa)}{I_{0}(\kappa)} .
$$

Similarly, the spatial recurrence rate, i.e., the number of oscillator pairs, which possesses the phase difference smaller than $l$, can also be computed analytically as

$$
\begin{aligned}
\operatorname{RR}(l, \kappa) & =\int_{0}^{2 \pi} \int_{-l}^{l} \rho_{\kappa}(\phi) \rho_{\kappa}(\phi+\eta) d \eta d \phi \\
& =\frac{2}{\pi I_{0}^{2}(\kappa)} \int_{0}^{l / 2} I_{0}(2 \kappa \cos \eta) d \eta .
\end{aligned}
$$

Figure 6(a) illustrates the behaviour of $r(\kappa)$ and $\mathrm{RR}(\kappa)$ given by expressions (10) and (11) for different values of the threshold $l$. Figure 6(b) shows a comparison of the numerical and theoretical dependence of RR on $r$. In the graph, the coupling parameter $\varepsilon$ acts as a parametrisation of the numerical results and the parameter $\kappa$ of the von Mises distribution for the theoretical results. The reason for the good agreement between analytical and numerical results can be explained by the fact that the phase distributions are normal to a good approximation. A similar situation was previously observed in oscillatory neural networks with spike timing-dependent plasticity. ${ }^{33}$

We remark that without coupling $\kappa=0$, the recurrence rate is $\operatorname{RR}(l, 0)=l / \pi$, which, in particular, implies that the phase of an oscillator will be closer than $l$ with $\bar{v}=N l / \pi$ other oscillators (expected value). As a result, in order to avoid calculating such a "spurious" synchronisation in the laminarity inspired coefficient (6), we choose the threshold $v_{\min }=N l / 2$, which is larger than $\bar{v}$, but still linearly proportional to $l$ and $N$.

\section{NETWORK OF NETWORKS}

To better mimic the brain structure, we build a network of networks consisting of four coupled small-world subnetworks, where each subnetwork has 100 neurons $(N=400)$. Figure 7 shows the adjacency matrix (pairs of connected neurons in black points) for the coupled small-world networks, 

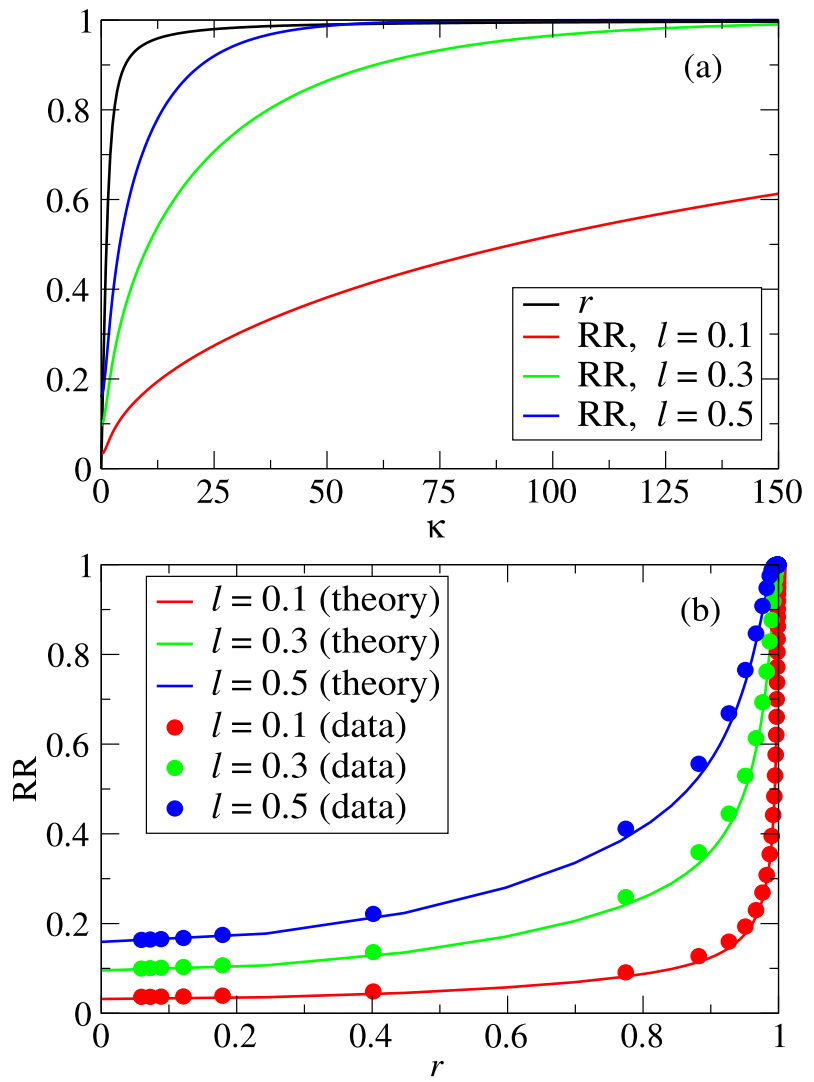

FIG. 6. (a) Analytical order parameter and spatial recurrence rate versus $\kappa$ for the random phases distributed according to the circular normal distribution [Eq. (9)] for different threshold values $l$. (b) Comparison of the numerical results with the theoretical for normally distributed phases. Spatial recurrence rate versus order parameter are shown for three different values of the threshold $l$. Lines are theoretical values and points correspond to numerical results.

where there are (intra) connections between neurons inside the small-world subnetwork and (inter) connections between neurons located in different subnetworks. We consider the probability of connections between neurons in the same subnetwork

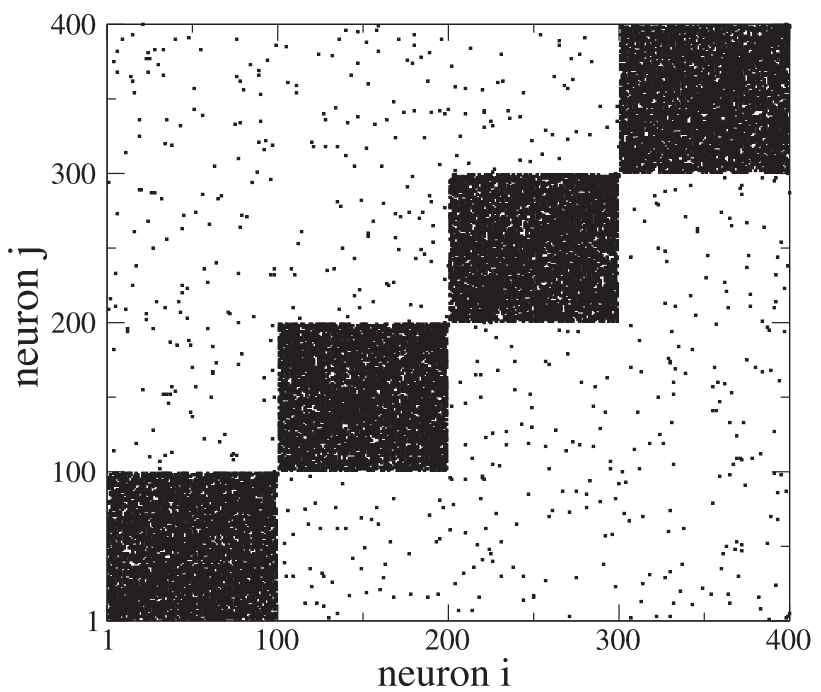

FIG. 7. Adjacency matrix of the network with four coupled small-world subnetworks for $p_{\text {intra }}=0.5$ and $p_{\text {inter }}=0.005$.
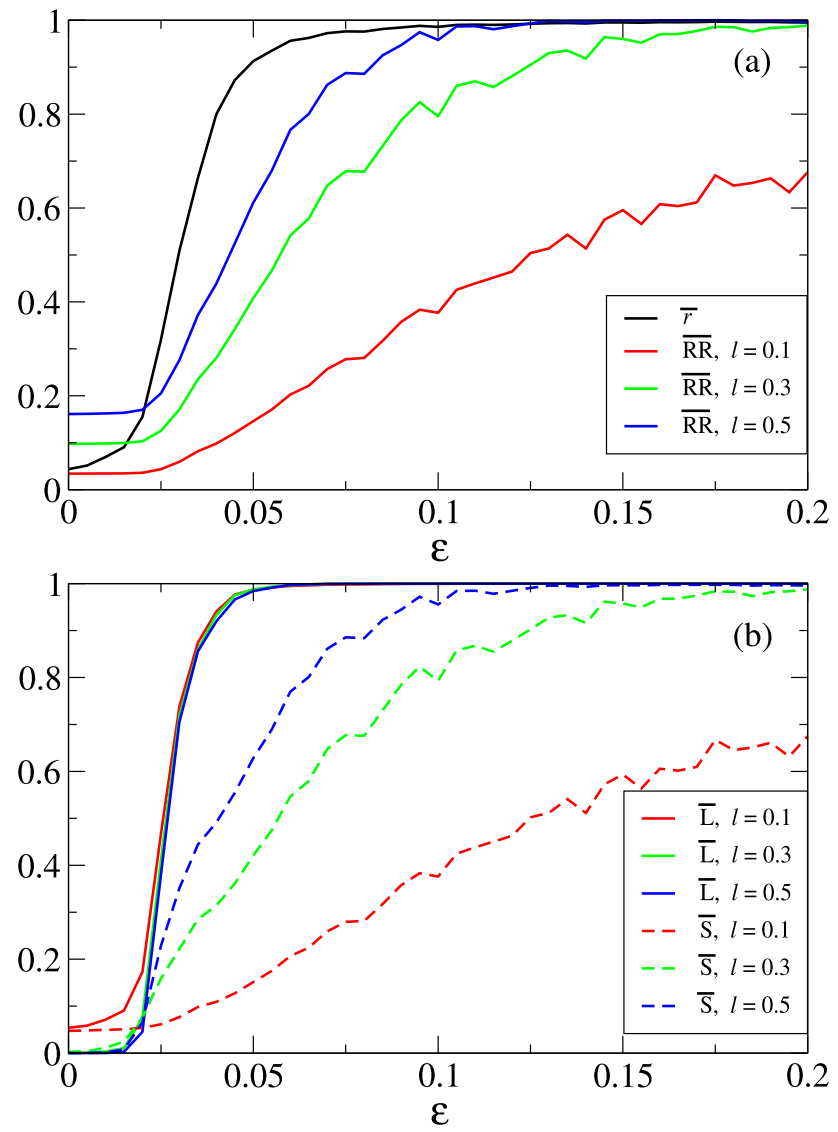

FIG. 8. (a) $\bar{r}$ and $\overline{\mathrm{RR}}$ as a function of $\varepsilon$ for $l=0.1$ (red), $l=0.3$ (green), and $l=0.5$ (blue). (b) $\overline{\mathrm{L}}$ and $\overline{\mathrm{S}}$ as a function of $\varepsilon$ for different $l$ values.

to be $p_{\text {intra }}=0.5$ and between different subnetworks $p_{\text {inter }}=$ 0.005 .

Figure 8 shows the collective dynamics measurements for our clustered network. This network exhibits phase synchronisation due to the fact that $\bar{r}$ goes to values about 1 [Fig. 8(a)]. We also verify that $\overline{\mathrm{RR}}$ varies for different $l$ [Fig. 8(a)] and it behaves like $\bar{S}$ [Fig. 8(b)]. The values of $\bar{L}$ follow approximately the same curves for different $l$, due to the fact that $v_{\min }$ changes with $l$.

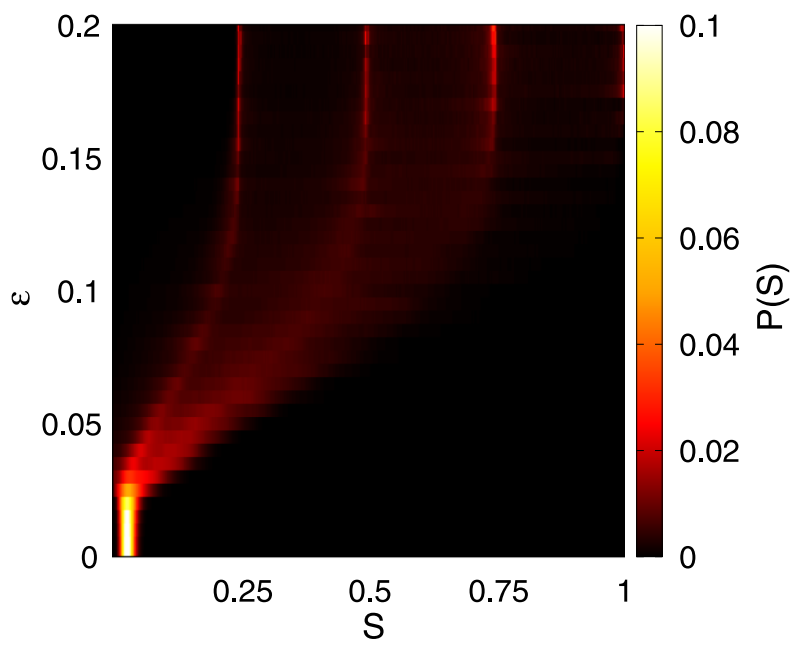

FIG. 9. Probability $P$ of finding a cluster of size $S$ depending on the coupling strength $\varepsilon$ and size $S$ for $l=0.1$. 
According to Fig. 9, for $l=0.1$, there are only groups of small size of synchronised neurons for $\varepsilon<0.025$. Similar results were obtained for one network, as it is shown in Fig. 5. However, when $\varepsilon$ is increased, for $\varepsilon>0.15$, we find larger synchronised groups. $\mathrm{P}(\mathrm{S})$ has significant values for $\mathrm{S}$ equal to $0.25,0.5,0.75$, and 1 , which are related to synchronised groups with the sizes $100,200,300$, and 400 , respectively. We can associate these values with the synchronisation inside and between the subnetworks. There are experimental evidences that phase synchronisation occurs between separated regions of the brain. ${ }^{34}$ Phase synchronisation between brain regions plays an important role in understanding memory processes. ${ }^{35}$

\section{CONCLUSIONS}

We have considered two types of neural networks composed of coupled Rulkov neurons: (i) small-world network and (ii) coupled small-world subnetworks. When the coupling strength was increased, we observed neural phase synchronisation of bursts. Depending on the coupling strength value, the clustered network can exhibit a coexistence of synchronous and desynchronous behaviours.

For the single small-world network, we verify that $\overline{R R}$ depends on $l$ and increases, similar to $\overline{\mathrm{S}}$, when $\varepsilon$ increases. During the transition to synchronisation, the phase distributions are shown to be close to circular normal distribution. This allows one to obtain certain analytical estimations of the dependence of the spatial recurrence rate on the order parameter. Close to synchronisation, for larger $\varepsilon$, the values $\mathrm{P}(\mathrm{S})$ indicate the appearance of larger synchronous groups of neurons.

We show that RQA can be also used for the identification of phase synchronisation in network of networks. Considering a network with four coupled small-world subnetworks, we observe that the size probability $\mathrm{P}(\mathrm{S})$ displays four maxima for larger values of $\varepsilon$, and these maxima can be related to synchronisation among the subnetworks.

In summary, RQA can be used as an efficient tool to study the transition to phase synchronisation in networks. In this article, we showed that RQA can be applied also for the analysis of synchronisation in networks composed of bursting elements. We believe that similar observations can appear for other models, since a phase can be associated with oscillatory behaviours. For instance, the spatial recurrence rate was used to identify chimera-like states in a network composed of coupled Hindmarsh-Rose neurons. ${ }^{16}$ More studies considering other models are necessary in order to extend the observed results to larger classes of systems.

In future works, we plan to use RQA to identify neural phase synchronisation in network of networks considering the structural connection matrix of the human brain. We also plan to investigate synchronisation by means of RQA in networks with neural plasticity, due to the fact that RQA allows one to find not only synchronous behaviours but also synchronised structures.

\section{ACKNOWLEDGMENTS}

We wish to acknowledge the support from CNPq, CAPES, Fundação Araucária, and São Paulo Research
Foundation (processes FAPESP 2011/19296-1, 2015/07311-7, 2016/23398-8, 2017/13502-5, 2017/20920-8, 2017/18977-1). This research was supported by Grant No. 2015/50122-0 from São Paulo Research Foundation (FAPESP) and DFG-IRTG Grant No. 1740/2.

${ }^{1}$ G. Deco, A. Buehlmann, T. Masquelier, and E. Hugues, "The role of rhythmic neural synchronization in rest and task conditions," Front. Hum. Neurosci. 5, 1-6 (2011).

${ }^{2}$ T. Womelsdorf, J.-M. Schoffelen, R. Oostenveld, W. Singer, R. Desimone, A. K. Engel, and P. Fries, "Modulation of neuronal interactions through neuronal synchronization," Science 316, 1609-1612 (2007).

${ }^{3}$ P. J. Uhlhaas and W. Singer, "Neural synchrony in brain disorders: Relevance for cognitive dysfunctions and pathophysiology," Neuron. 52, 155-168 (2006).

${ }^{4}$ L. L. Rubchinsky, C. Park, and R. M. Worth, "Intermittent neural synchronization in Parkinson's disease," Nonlinear Dyn. 68, 329-346 (2012).

${ }^{5}$ K. Lehnertz, S. Bialonski, M.-T. Horstmann, D. Krug, A. Rothkegel, M. Staniek, and T. Wagner, "Synchronization phenomena in human epileptic brain networks," J. Neurosci. Methods 183, 42-48 (2009).

${ }^{6}$ S. Boccaletti, G. Bianconi, R. Criado, C. I. del Genio, J. Gómez-Gardeñes, M. Romance, I. Sendiña-Nadal, Z. Wang, and M. Zanin, "The structure and dynamics of multilayer networks," Phys. Rep. 544, 1-22 (2014).

${ }^{7}$ D. Y. Kenett, M. Perc, and S. Boccaletti, "Networks of networks-An introduction," Chaos Solitons Fractals 80, 1-6 (2015).

${ }^{8}$ N. F. Rulkov, "Modeling of spiking-bursting neural behavior using twodimensional map," Phys. Rev. E 65, 041922 (2001).

${ }^{9}$ D. J. Watts and S. H. Strogatz, "Collective dynamics of 'small-world' networks," Nature 393, 440 (1998).

${ }^{10}$ O. Sporns and C. J. Honey, "Small worlds inside big brains," Proc. Natl. Acad. Sci. 103, 19219 (2006).

${ }^{11}$ S. Achard, R. Salvador, B. Whitcher, J. Suckling, and E. Bullmore, "A resilient, low-frequency, small-world human brain functional network with highly connected association cortical hubs," J. Neurosci. 26, 63 (2006).

${ }^{12}$ E. L. Lameu, F. S. Borges, R. R. Borges, A. M. Batista, M. S. Baptista, and R. L. Viana, "Network and external perturbation induce burst synchronisation in cat cerebral cortez," Commun. Nonlinear Sci. Numer. Simul. 34, 45 (2016).

${ }^{13}$ E. L. Lameu, F. S. Borges, R. R. Borges, K. C. Iarosz, I. L. Caldas, A. M. Batista, R. L. Viana, and J. Kurths, "Supression of phase synchronisation in network based on cat's brain," Chaos 26, 043107 (2016).

${ }^{14}$ N. Marwan, M. C. Romano, M. Thiel, and J. Kurths, "Recurrence plots for the analysis of complex systems," Phys. Rep. 438, 237 (2007).

${ }^{15}$ M. S. Santos, J. D. Szezech, Jr., A. M. Batista, I. L. Caldas, R. L. Viana, and S. R. Lopes, "Recurrence quantification analysis of chimera states," Phys. Lett. A 379, 2188 (2015).

${ }^{16}$ M. S. Santos, J. D. Szezech, Jr., F. S. Borges, K. C. Iarosz, I. L. Caldas, A. M. Batista, R. L. Viana, and J. Kurths, "Chimera-like states in a neuronal network model of the cat brain," Chaos Solitons Fractals 101, 86 (2017).

${ }^{17}$ J. P. Eckmann, S. O. Kamphorst, and D. Ruelle, "Recurrence plot of dynamical systems," Europhys. Lett. 5, 973 (1987).

${ }^{18}$ D. B. Vasconcelos, S. R. Lopes, R. L. Viana, and J. Kurths, "Spatial recurrence plots," Phys. Rev. E 73, 1-10 (2006).

${ }^{19}$ N. Marwan, N. Wessel, U. Meyerfeldt, A. Schirdewan, and J. Kurths, "Recurrence-plot-based measures of complexity and their application to heart-rate-variability data," Phys. Rev. E 66, 1-8 (2002).

${ }^{20}$ C. A. S. Batista, A. M. Batista, J. A. C. de Pontes, R. L. Viana, and S. R. Lopes, "Chaotic phase synchronization in scale-free networks of bursting neurons," Phys. Rev. E 76, 016218 (2007).

${ }^{21}$ R. L. Viana, A. M. Batista, C. A. S. Batista, J. C. A. de Pontes, F. A. dos S. Silva, and S. R. Lopes, "Bursting synchronization in networks with longrange coupling mediated by a diffusing chemical substance," Commun. Nonlinear Sci. Numer. Simul. 17, 2924 (2012).

${ }^{22}$ Y. Kuramoto, Oscillations, Waves, and Turbulence (Springer-Verlag, Berlin, 1984).

${ }^{23}$ J. M. V. Grzybowski, E. E. N. Macau, and T. Yoneyama, "On synchronization in power-grids modelled as networks of second-order Kuramoto oscillators," Chaos 26, 113113 (2016).

${ }^{24}$ C. Freitas, E. Macau, and R. L. Viana, "Synchronization versus neighborhood similarity in complex networks of nonidentical oscillators," Phys. Rev. E 92, 032901 (2015). 
${ }^{25}$ C. Freitas, E. Macau, and A. Pikovsky, "Partial synchronization in networks of non-linearly coupled oscillators: The Deserter Hubs Model," Chaos 25, 043119 (2015).

${ }^{26}$ D. Witthaut and M. Timme, "Kuramoto dynamics in Hamiltonian systems," Phys. Rev. E 90, 032917 (2014).

${ }^{27}$ C. A. S. Batista, S. R. Lopes, R. L. Viana, and A. M. Batista, "Delayed control of bursting synchronization in a scale-free neuronal network," Neural Netw. 23, 114 (2010).

${ }^{28}$ J. P. Zbilut and C. L. Webber, Jr., "Embeddings and delays as derived from quantification of recurrence plots," Phys. Lett. A 171, 199 (1992).

${ }^{29}$ J. P. Zbilut, N. Thomasson, and C. L. Webber, "Recurrence quantification analysis as a tool for nonlinear exploration of nonstationary cardiac signals," Med. Eng. Phys. 24, 53 (2002).

${ }^{30}$ Z. O. Guimarães-Filho, I. L. Caldas, R. L. Viana, J. Kurths, I. C. Nascimento, and Yu. K. Kuznetsov, "Recurrence quantification analysis of electrostatic fluctuations in fusion plasmas," Phys. Lett. A 372, 1088 (2008).

${ }^{31}$ M. E. J. Newman and D. J. Watts, "Renormalization group analysis of the small-world network model," Phys. Lett. A 263, 341 (1999).

${ }^{32}$ B. Sonnenschein and L. Schimansky-Geier, "Approximate solution to the stochastic Kuramoto model," Phys. Rev. E 88, 052111 (2013).

${ }^{33}$ V. Popovych, S. Yanchuk, and P. A. Tass, "Self-organized noise resistance of oscillatory neural networks with spike timing-dependent plasticity," Sci. Rep. 3, 2926 (2013).

${ }^{34}$ L. Melloni, C. Molina, M. Pena, D. Torres, W. Singer, and E. Rodriguez, "Synchronization of neural activity across cortical areas correlates with conscious perceptions," J. Neurosci. 27, 2858 (2007).

${ }^{35} \mathrm{~J}$. Fell and N. Axmacher, "The role of phase synchronization in memory processes," Nat. Rev. Neurosci. 12, 105 (2011). 